\title{
Estimation of Wellbore and Formation Temperatures during the Drilling Process under Lost Circulation Conditions
}

\author{
Mou Yang, ${ }^{1}$ Yingfeng Meng, ${ }^{1}$ Gao Li, ${ }^{1}$ Yongjie Li, ${ }^{1}$ Ying Chen, \\ Xiangyang $\mathrm{Zhao}^{2}$, and Hongtao $\mathrm{Li}^{1}$ \\ ${ }^{1}$ State Key Laboratory of Oil and Gas Reservoir Geology and Exploitation, Southwest Petroleum University, Chengdu 610500, China \\ ${ }^{2}$ Research Institute of Petroleum Engineering, SINOPEC, Beijing 100101, China
}

Correspondence should be addressed to Yingfeng Meng; cwctmyf@vip.sina.com and Gao Li; 546652841@qq.com

Received 4 February 2013; Revised 22 June 2013; Accepted 24 June 2013

Academic Editor: Zhijun Zhang

Copyright (C) 2013 Mou Yang et al. This is an open access article distributed under the Creative Commons Attribution License, which permits unrestricted use, distribution, and reproduction in any medium, provided the original work is properly cited.

Significant change of wellbore and surrounding formation temperatures during the whole drilling process for oil and gas resources often leads by annulus fluid fluxes into formation and may pose a threat to operational security of drilling and completion process. Based on energy exchange mechanisms of wellbore and formation systems during circulation and shut-in stages under lost circulation conditions, a set of partial differential equations were developed to account for the transient heat exchange process between wellbore and formation. A finite difference method was used to solve the transient heat transfer models, which enables the wellbore and formation temperature profiles to be accurately predicted. Moreover, heat exchange generated by heat convection due to circulation losses to the rock surrounding a well was also considered in the mathematical model. The results indicated that the lost circulation zone and the casing programme had significant effects on the temperature distributions of wellbore and formation. The disturbance distance of formation temperature was influenced by circulation and shut-in stages. A comparative perfection theoretical basis for temperature distribution of wellbore-formation system in a deep well drilling was developed in presence of lost circulation.

\section{Introduction}

Annulus fluid fluxed into formation usually in presence of lost circulation problem occurs in oil-gas and geothermal wells during the drilling stage with increasing well depth, thus resulting in continuous variation of the temperature of wellbore (inside drilling string fluid, drilling pipe, and annulus) and surrounding formation (casing, cement sheath, static drilling fluid, and rock). Moreover, the determination of transient temperature distributions in and around oil-gas well under circulation and shut-in conditions is a complex task because of the occurrence of lost circulation due to the change of drilling fluid flow state and formation property [1-3]. Therefore, it is important to obtain the accurate temperature distributions of wellbore and formation under lost circulation conditions, which can develop the adequate drilling style and design the excellent property of drilling fluids and cement slurries $[4,5]$.
A reliable and accurate estimation of such temperature distribution requires a complete dynamic thermal study related to the drilling fluid flow in and around the wellbore, which includes a set of numerical models as well as boundary and initial conditions. At present, the estimation temperature method in and around oil-gas well is mainly classified into two classes. One deals with using classical analytical methods based on conductive heat flow in cylindrical coordinate [611], exclusive of conductive-convective heat flow method [12] and the spherical and radial heat flow method [13]. These models have been considered as excellent methods in many applications due to their simplicity, whereas the formation temperature obtained by these methods is normally lower than the initial temperature [14]. The other class attempts to describe the transient heat transfer processes using numerical models based on the energy balance principle in each region of a well during circulation and shut-in stages [15-19]. 
The previous estimation methods are mainly focused on studying the wellbore and formation temperatures under no lost circulation condition. That is to say, these methods cannot accurately estimate the wellbore and formation temperatures in presence of lost circulation. Recently, with regard to this, only a few studies involved several aspects for estimating the temperatures of fluid and formation when lost circulation is being [20-22]. However, those studies have little attention on studying the heat exchanged mechanism and law between wellbore and formation under lost circulation conditions by the numerical model.

Therefore, in this work, the development of transient heat transfer model for estimation of wellbore and formation temperatures in oil-gas wells during circulation and shutin stages under lost circulation condition are presented. Here, the well-formation interface is considered as a porous medium through which fluid lost by circulation [23]. Moreover, to deeply analyze lost circulation process for radial heat transfer equation, the model also takes the radial fluid motion and the radial heat flow from annulus to formation into consideration. Thereby, under lost circulation, the comprehensive model is applied to estimate each heat transfer region in a well according to the actual data of well drilling.

\section{Physical Model}

The physical model of lost circulation during circulation stage is shown in Figure 1. The process of circulation is considered as three distinct phases: (1) fluid enters the drill pipe with a specified temperature $\left(T_{\text {in }}\right)$ at the surface and passes down with flow velocity $v_{z 1}$ in the $z$ direction; (2) fluid exits the drill pipe through the bit and enters the annulus at the bottom; (3) fluid passes up the annulus with flow velocity $v_{z 3}$ and exits the annulus with a specified temperature $\left(T_{\text {out }}\right)$ at the surface $[15,18]$. If lost circulation is being in a certain formation, drilling fluid would be flown into surrounding formation so that it becomes hard to precisely define the temperature profile of a well. Therefore, to simulate thermal behavior of fluid during the circulation process, it is necessary to develop a set of partial differential equations under the actual casing program and drill string assembly conditions, which is illustrated in Figure 1. Mass and energy conservation is considered as incompressible flow in the axial $(z)$ and radial $(r)$ directions. Meanwhile, the effect factors of boundary conditions among each control unite are taken into account in the solving model.

During the circulation stage, the fluid passes down the pipe in the $z$ direction, and its temperature distribution is determined by the rate of heat convection down the drilling pipe and heat exchange with the metallic pipe wall. At the bottom, the fluid temperature at the outlet of the drill pipe is the same as the fluid temperature at the entrance of the annulus, and then the fluid keeps on flowing up in the annulus. Similarly, the annulus fluid temperature is determined by the rate of heat convection up the annulus, the rate of heat exchange between the annulus and drill pipe wall, and the rate of heat exchange between the wall of the well and annulus fluid [23]. Meanwhile, well depth and flow rate of lost circulation have great effects on annulus temperature. In addition, fluid friction, rotational energy, and drill bit energy also have significant influence on the overall energy balance of the wellbore [24]. During shut-in stage, above the lost depth, all wellbore fluid will flow into formation. Therefore, the temperatures in the wellbore which are decided by fluid flow state depend upon a number of different thermal processes involving conductive and convective mechanisms in different sections of well. When wellbore fluid is in flowing state, the fluid temperatures of inside drill string and annulus are strong dependent upon the rate of lost circulation in heat convection way; if all of wellbore fluid above the loss depth flow into formation, the heat exchange between wellbore and formation is only in a conduction way.

\section{Mathematical Model}

3.1. Assumption Conditions. The mathematical model consisted of a set of partial differential equations used to describe the temperatures of wellbore and formation. The fundamental assumptions of numerical model include the following.

(i) Each control unit of wellbore and formation system is cylindrical geometry.

(ii) The physical properties of the formation, cement, and metal pipe are constant with the change of depths [5]. The parameters include thermal conductivity, density, heat capacity, and viscosity.

(iii) The radial temperature gradient within the fluid may be neglected.

(iv) The heat conduction equation through surrounding wellbore is solved by using a two-dimensional transient axial-symmetric temperature distribution.

(v) Viscous dissipation and thermal expansion effects are neglected.

Under these conditions, the governing equations and initial and boundary conditions for each region are as follows.

\subsection{Mathematical Formulation}

\subsubsection{Transient Heat Transfer during Circulation Stage}

(1) Inside the Drilling String. The numerical model which can calculate the temperature distribution of inside drilling string is complemented by the following three considerations: (1) the inlet fluid temperature is the boundary condition of the model; (2) the flow velocity of fluid in the $z$ direction is also defined by mass flow rate; and (3) heat generated by fluid fraction. Therefore, based on energy conservation principle, the model is expressed as follows:

$$
\frac{Q_{m}}{\pi r_{1}^{2}}-\frac{\partial\left(\rho_{1} c_{1} T_{1} q\right)}{\pi r_{1}^{2} \partial z}-\frac{2 h_{1}\left(T_{1}-T_{2}\right)}{r_{1}}=\frac{\partial\left(\rho_{1} c_{1} T_{1}\right)}{\partial t}
$$




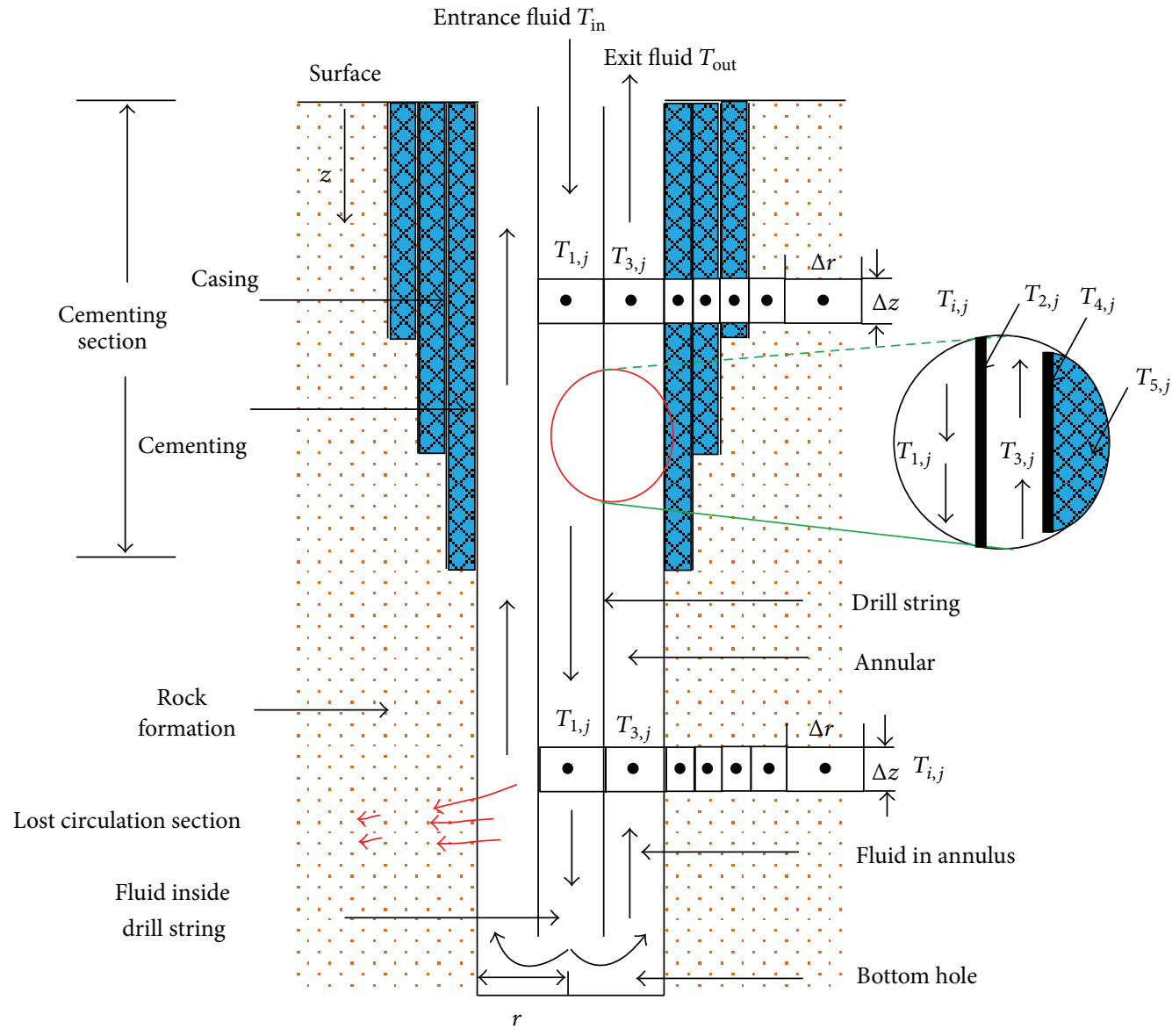

FIgURE 1: Physical model of drilling fluid circulation under lost circulation condition.

The boundary condition between fluid and inner wall of drill string is written as follows:

$$
-\lambda_{2}\left(\frac{\partial T_{2}}{\partial r}\right)_{r=r_{1}}=h_{1}\left(T_{2}-T_{1}\right)
$$

where $T_{1}, T_{2}$ are the temperatures of inside drilling string fluid and drilling string wall, respectively; $Q_{m}$ is the energy source term of unit length inside the drilling string; $\rho_{1}$ is the density of drilling fluid; $q$ is the flow rate of inside drill string; $c_{1}$ is the specific heat capacity of drilling fluid; $r_{1}$ is the radius of inside drill string; $\lambda_{2}$ is the thermal conductivity of drill string; and $h_{1}$ is the convection coefficient of inside drilling string wall.

(2) Drill String Wall. The formulation calculates the temperature distribution of drill string wall, and the conditions here are defined by the three sections: (1) the mass flow rate of fluid in the inside drill string and annulus; (2) the vertical conduction of heat in the drill pipe; and (3) the radial exchange of heat between the drill pipe and the fluid inside and outside the string. The numerical model of the drill string wall is given as follows:

$$
\begin{gathered}
\frac{\partial}{\partial z}\left(\lambda_{2} \frac{\partial T_{2}}{\partial z}\right)+\frac{2 r_{1} h_{1}}{\left(r_{2}^{2}-r_{1}^{2}\right)}\left(T_{1}-T_{2}\right) \\
-\frac{2 r_{2} h_{2}}{\left(r_{2}^{2}-r_{1}^{2}\right)}\left(T_{2}-T_{3}\right)=\frac{\partial\left(\rho_{2} c_{2} T_{2}\right)}{\partial t} .
\end{gathered}
$$

The boundary condition influenced the temperature distribution of drill string wall includes two parts: one is the heat exchange between fluid of inside drill string and drill string, which is expressed by (2), and the other is heat exchange between annulus and the drill string which is written as

$$
-\lambda_{2}\left(\frac{\partial T_{2}}{\partial r}\right)_{r=r_{2}}=h_{2}\left(T_{2}-T_{3}\right),
$$

where $T_{3}$ is the temperature of annulus fluid; $\rho_{2}$ is the density of drill string wall; $c_{2}$ is the specific heat capacity of drill string; $r_{2}$ is the outer radius of drill string; $\lambda_{2}$ is the thermal conductivity of drill string; and $h_{2}$ is the convection coefficient of outer drilling string wall.

(3) In the Annulus. The factors that influenced the annulus temperature are consisted of three sections: (1) the mass flow 
rate of fluid; (2) the temperature distributions of drill string and wellbore walls; and (3) heat generated by fluid fraction and drilling string rotation. The transient heat transfer model in the annulus is expressed as follows:

$$
\begin{aligned}
& \frac{\partial\left(\rho_{3} c_{3} q^{\prime} T_{3}\right)}{\pi\left(r_{3}^{2}-r_{2}^{2}\right) \partial z}+\frac{2 r_{2} h_{2}\left(T_{2}-T_{3}\right)}{r_{3}^{2}-r_{2}^{2}}-\frac{2 r_{3} h_{\mathrm{ef}}\left(T_{3}-T_{4}\right)}{r_{3}^{2}-r_{2}^{2}} \\
& \quad+\frac{Q_{a}}{\pi\left(r_{3}^{2}-r_{2}^{2}\right)}=\frac{\partial\left(\rho_{3} c_{3} T_{3}\right)}{\partial t} .
\end{aligned}
$$

The interface between annulus and wellbore wall is important since it mathematically couples the surrounding formation with the flow in the annulus. Therefore, to guarantee continuity of heat flux during circulation and shut-in condition, the boundary conditions are

$$
-\lambda_{3}\left(\frac{\partial T_{3}}{\partial r}\right)_{r=r_{3}}+h_{\mathrm{ef}}\left(T_{4}-T_{3}\right)=\lambda_{\mathrm{ef}}\left(\frac{\partial T_{4}}{\partial r}\right)_{r=r_{3}},
$$

where $T_{4}$ is the interface temperature between annulus fluid and borehole wall; $Q_{a}$ is the energy source term of unit length inside annulus; $q^{\prime}$ is the flow rate of annulus; $r_{3}$ is the radius of borehole wall; $\lambda_{3}$ is the thermal conductivity of annulus fluid; effective thermal conductivity $\lambda_{\text {ef }}$ considers the effect of porosity; $h_{3}$ is the convection coefficient of borehole wall; $h_{\mathrm{ef}}$ is the effective heat transfer coefficient which considers the effect porosity.

(4) Each Heat Transfer Region in Surrounding Wellbore. The effect of factor on heat exchange for the surrounding wellbore includes four sections: (1) the vertical conduction of heat in the medium; (2) the rate of heat exchange among volume elements; and (3) the rate of heat exchange for formation fluid which can flow in the porous medium. The energy balance in each heat transfer medium is

$$
\begin{gathered}
\frac{\partial}{\partial z}\left(\lambda_{\mathrm{ef}} \frac{\partial T_{i}}{\partial z}\right)+\frac{1}{r_{i}} \frac{\partial}{\partial r}\left(\lambda_{\mathrm{ef}} r_{i} \frac{\partial T_{i}}{\partial r}\right) \\
=\left(\rho c_{p}\right)_{\mathrm{ef}}\left(\frac{\partial T_{i}}{\partial t}+v_{r} \frac{\partial T_{i}}{\partial r}\right),
\end{gathered}
$$

where

$$
\begin{gathered}
\left(\rho c_{p}\right)_{\mathrm{ef}}=\phi\left(\rho c_{p}\right)_{s}+(1-\phi)\left(\rho c_{p}\right)_{l} \\
v_{r}=f\left(\phi, m, m_{f u}, \rho_{\mathrm{in}}, A_{l}\right) .
\end{gathered}
$$

The mathematical formulation for the hydrodynamic model of rock formation is based on one-dimensional volume-averaging equations that govern the hydrodynamic phenomena of an incompressible fluid across an isotropic porous medium [22], which are represented as follows:

$$
\begin{gathered}
v_{r}=-\frac{K}{\mu} \frac{\partial p}{\partial r} \\
\frac{K_{l}}{\mu}\left(\frac{\partial^{2} p}{\partial r^{2}}+\frac{1}{r} \frac{\partial p}{\partial r}\right)+\frac{\widetilde{q}}{\rho_{\text {in }}}=0,
\end{gathered}
$$

where $T_{i}$ is different unit temperature of porous medium in the formation; $r_{i}$ is the radius of porous medium in the formation; the magnitude of $i$ is decided by casing program $(i \geq 4) ; \phi$ is an effective porosity of formation; $s$ and $l$ represent rock and pore fluid, respectively; $v_{r}$ is the radial flow velocity; $m_{\mathrm{fu}}$ is formation fluid mass flow to annulus; $m$ is the drilling fluid of mass flow; $A_{l}$ is the lateral flow area; $\mu$ is the dynamic viscosity; $p$ is the intrinsic average pressure; $K$ is the absolute permeability of the isotropic porous medium; $\widetilde{q}$ is the mass source term; and $K_{l}$ is the relative permeability.

3.2.2. Transient Heat Transfer during Shut-In Stage. During stop circulation stage, the heat exchange method can be classified into two ways, which is dependent on the interface between gas and liquid. Therefore, combined with the study of heat exchange mechanism between wellbore and formation during fluid circulation stage and energy and mass conservation principles, the description of heat exchange types during shut-in stage is presented by a set of partial difference equations.

\section{(1) In the Drill String}

(1) The transient heat transfer model of above interface between gas and liquid and below lost formation is expressed as

$$
\begin{gathered}
\frac{2 \lambda_{1}^{\prime} \lambda_{2}^{\prime}\left(T_{2}^{\prime}-T_{1}^{\prime}\right)}{\lambda_{2}^{\prime} r_{1}^{2} \ln \left[\left(r_{1}+r_{2}\right) / 2 r_{1}\right]+\lambda_{1}^{\prime} r_{1}^{2} \ln \left[2 r_{2} /\left(r_{1}+r_{2}\right)\right]} \\
=\frac{\partial\left(\rho_{1}^{\prime} c_{1}^{\prime} T_{1}^{\prime}\right)}{\partial t} .
\end{gathered}
$$

(2) The transient heat transfer model of below interface between gas and liquid and above lost formation is described as

$$
\frac{Q_{m}^{\prime}}{\pi r_{1}^{2}}-\frac{\partial\left(\rho_{1}^{\prime} c_{1}^{\prime} q^{\prime} T_{1}^{\prime}\right)}{\pi r_{1}^{2} \partial z}-\frac{2 h_{1}^{\prime}\left(T_{1}^{\prime}-T_{2}^{\prime}\right)}{r_{1}}=\frac{\partial\left(\rho_{1}^{\prime} c_{1}^{\prime} T_{1}^{\prime}\right)}{\partial t} \text {. }
$$

\section{(2) Drill String Wall}

(1) The transient heat transfer model of above interface between gas and liquid and below lost formation is expressed as

$$
\begin{aligned}
& \frac{2 \lambda_{3}^{\prime} \lambda_{2}^{\prime}\left(T_{3}^{\prime}-T_{2}^{\prime}\right)}{\left(\lambda_{3}^{\prime} \ln \left(\left(r_{1}+r_{2}\right) / 2 r_{1}\right)+\lambda_{2}^{\prime} \ln \left(2 r_{2} /\left(r_{1}+r_{2}\right)\right)\right)\left(r_{1}^{2}-r_{0}^{2}\right)} \\
& +\lambda_{2}^{\prime} \frac{\partial^{2} T_{2}^{\prime}}{\partial z^{2}} \\
& \quad-\frac{2 \lambda_{1}^{\prime} \lambda_{2}^{\prime}\left(T_{2}^{\prime}-T_{1}^{\prime}\right)}{\left(\lambda_{2}^{\prime} \ln \left(\left(r_{0}+r_{1}\right) / 2 r_{0}\right)+\lambda_{1}^{\prime} \ln \left(2 r_{1} /\left(r_{0}+r_{1}\right)\right)\right)\left(r_{1}^{2}-r_{0}^{2}\right)} \\
& =\frac{\partial\left(\rho_{2}^{\prime} c_{2}^{\prime} T_{2}^{\prime}\right)}{\partial t} .
\end{aligned}
$$


(2) The transient heat transfer model of below interface between gas and liquid and above lost formation is expressed as

$$
\begin{gathered}
\frac{\partial}{\partial z}\left(\lambda_{2}^{\prime} \frac{\partial T_{2}^{\prime}}{\partial z}\right)+\frac{2 r_{1} h_{1}^{\prime}}{\left(r_{2}^{2}-r_{1}^{2}\right)}\left(T_{1}^{\prime}-T_{2}^{\prime}\right) \\
-\frac{2 r_{2} h_{2}^{\prime}}{\left(r_{2}^{2}-r_{1}^{2}\right)}\left(T_{2}^{\prime}-T_{3}^{\prime}\right)=\frac{\partial\left(\rho_{2}^{\prime} c_{2}^{\prime} T_{2}^{\prime}\right)}{\partial t} .
\end{gathered}
$$

\section{(3) In the Annulus}

(1) The transient heat transfer model of above interface between gas and liquid and below lost formation is expressed as

$$
\begin{aligned}
& \frac{2 \lambda_{3}^{\prime} \lambda_{\mathrm{ef}}^{\prime}\left(T_{4}^{\prime}-T_{3}^{\prime}\right)}{\left(\lambda_{\mathrm{ef}}^{\prime} \ln \left(\left(r_{2}+r_{3}\right) / 2 r_{2}\right)+\lambda_{3}^{\prime} \ln \left(2 r_{3} /\left(r_{2}+r_{3}\right)\right)\right)\left(r_{2}^{2}-r_{1}^{2}\right)} \\
& -\frac{2 \lambda_{3}^{\prime} \lambda_{2}^{\prime}\left(T_{3}^{\prime}-T_{2}^{\prime}\right)}{\left(\lambda_{3}^{\prime} \ln \left(\left(r_{1}+r_{2}\right) / 2 r_{1}\right)+\lambda_{2}^{\prime} \ln \left(2 r_{2} /\left(r_{1}+r_{2}\right)\right)\right)\left(r_{2}^{2}-r_{1}^{2}\right)} \\
& =\frac{\partial\left(\rho_{3}^{\prime} c_{3}^{\prime} T_{3}^{\prime}\right)}{\partial t} .
\end{aligned}
$$

(2) The transient heat transfer model of below interface between gas and liquid and above lost formation is described as

$$
\begin{aligned}
& \frac{\partial\left(\rho_{3}^{\prime} c_{3}^{\prime} q^{\prime \prime} T_{3}^{\prime}\right)}{\pi\left(r_{3}^{2}-r_{2}^{2}\right) \partial z}+\frac{2 r_{2} h_{2}^{\prime}\left(T_{2}^{\prime}-T_{3}^{\prime}\right)}{r_{3}^{2}-r_{2}^{2}}-\frac{2 r_{3} h_{\mathrm{ef}}^{\prime}\left(T_{3}^{\prime}-T_{4}^{\prime}\right)}{r_{3}^{2}-r_{2}^{2}} \\
& \quad+\frac{Q_{a}^{\prime}}{\pi\left(r_{3}^{2}-r_{2}^{2}\right)}=\frac{\partial\left(\rho_{3}^{\prime} c_{3}^{\prime} T_{3}^{\prime}\right)}{\partial t},
\end{aligned}
$$

where the meaning parameters of the shut-in stage defined from (10) to (15) are the same as that of circulation stage.

The form of transient numerical model for each heat transfer region surrounding wellbore during shut-in stage is also the same as (7).

\section{Numerical Solutions}

To obtain the temperature distribution on the term of time, the solution of these equations is complicated. Developed models incorporate solution methods which are based on finite difference techniques. The wellbore and the adjacent formation are represented by a two-dimensional, mesh grid including a number of radial elements due to casing program and a variable number of vertical elements depending on the well depth. Each of radial elements corresponds to different portion of the wellbore cross-section from inside drill string into the formation [21]. Therefore, a set of partial differential equations can be presented as finite difference form using finite differences technique for each element of grid so as to describe the transient heat exchange in each element for an implicit form [25]. A set of nonlinear algebraic equations are then solved using an iterative method until the error range can be accepted. The case of finite difference can be defined as follows. The spatial derivatives and the time derivatives are the first-order as exhibited in (16):

$$
\frac{\partial T}{\partial z} \approx \frac{T_{i, j}^{n+1}-T_{i, j-1}^{n+1}}{\Delta z_{j}} .
$$

The second-order spatial derivatives are represented by three-point central difference approximations [26, 27]:

$$
\frac{\partial^{2} T}{\partial z^{2}} \approx \frac{1}{\Delta z_{j}}\left(\frac{T_{i, j+1}^{n+1}-T_{i, j}^{n+1}}{\Delta z_{j+0.5}}-\frac{T_{i, j}^{n+1}-T_{i, j-1}^{n+1}}{\Delta z_{j-0.5}}\right) .
$$

The time discretization at node is expressed in

$$
\frac{\partial T}{\partial t} \approx \frac{T_{i, j}^{n+1}-T_{i, j}^{n}}{\Delta t} .
$$

Application of earlier definitions enables the equation for each node to be written in single generalized vector form:

$$
\alpha_{i j} T_{i-1, j}^{n+1}+\beta_{i j} T_{i, j}^{n+1}+\gamma_{i j} T_{i+1, j}^{n+1}+\delta_{i j} T_{i, j-1}^{n+1}+\lambda_{i j} T_{i, j+1}^{n+1}=C_{i j} .
$$

The matrix form of finite difference for each node is given:

$$
a \vec{T}^{n+1}=\vec{C}
$$

The finite difference equations are solved by fast successive overrelaxation (SOR) iteration method; the following general form for each node is expressed as follows:

$$
\begin{aligned}
T_{i, j}^{N+1}= & \frac{\omega}{B_{i, j}}[ \\
& F_{i, j} T_{i, j}^{N}+A_{i, j} T_{i-1, j}^{N+1} \\
& \left.+C_{i, j} T_{i+1, j}^{N+1}+D_{i, j} T_{i, j-1}^{N+1}+E_{i, j} T_{i, j+1}^{N+1}\right] \\
& +(1-\omega) \stackrel{v}{v+1} T_{i, j}^{N+1} .
\end{aligned}
$$

Using implicit form of finite difference method, (1) and (2) are, respectively, shown as follows:

$$
\begin{gathered}
-\frac{\rho_{1} q c_{1}}{\pi r_{1}^{2} \Delta z_{j}} \cdot T_{1, j-1}^{n+1}+\left(\frac{\rho_{1} c_{1}}{\Delta t}+\frac{\rho_{1} q c_{1}}{\pi r_{1}^{2} \Delta z_{j}}+\frac{2 h_{1}}{r_{1}}\right) \cdot T_{1, j}^{n+1} \\
-\frac{2 h_{1}}{r_{1}} \cdot T_{2, j}^{n+1}=\frac{Q_{1}}{\pi r_{1}^{2}}+\frac{\rho_{1} c_{1}}{\Delta t} \cdot T_{1, j}^{n} \\
\frac{2 r_{1} h_{1}}{\left(r_{2}^{2}-r_{1}^{2}\right)} \cdot T_{1, j}^{n+1}+\frac{\lambda_{2}}{\Delta z_{j} \Delta z_{j-0.5}} \cdot T_{2, j-1}^{n+1} \\
+\frac{\lambda_{2}}{\Delta z_{j} \Delta z_{j+0.5}} \cdot T_{2, j+1}^{n+1}+\frac{2 r_{2} h_{2}}{\left(r_{2}^{2}-r_{1}^{2}\right)} \cdot T_{3, j}^{n+1} \\
-\left(\frac{\lambda_{2}}{\Delta z_{j} \Delta z_{j-0.5}}+\frac{\lambda_{2}}{\Delta z_{j} \Delta z_{j+0.5}}+\frac{2 r_{1} h_{1}}{\left(r_{2}^{2}-r_{1}^{2}\right)}\right. \\
\left.+\frac{2 r_{2} h_{2}}{\left(r_{2}^{2}-r_{1}^{2}\right)}+\frac{\rho_{2} c_{2}}{\Delta t}\right) \cdot T_{2, j}^{n+1}=-\frac{\rho_{2} c_{2}}{\Delta t} \cdot T_{2, j}^{n},
\end{gathered}
$$




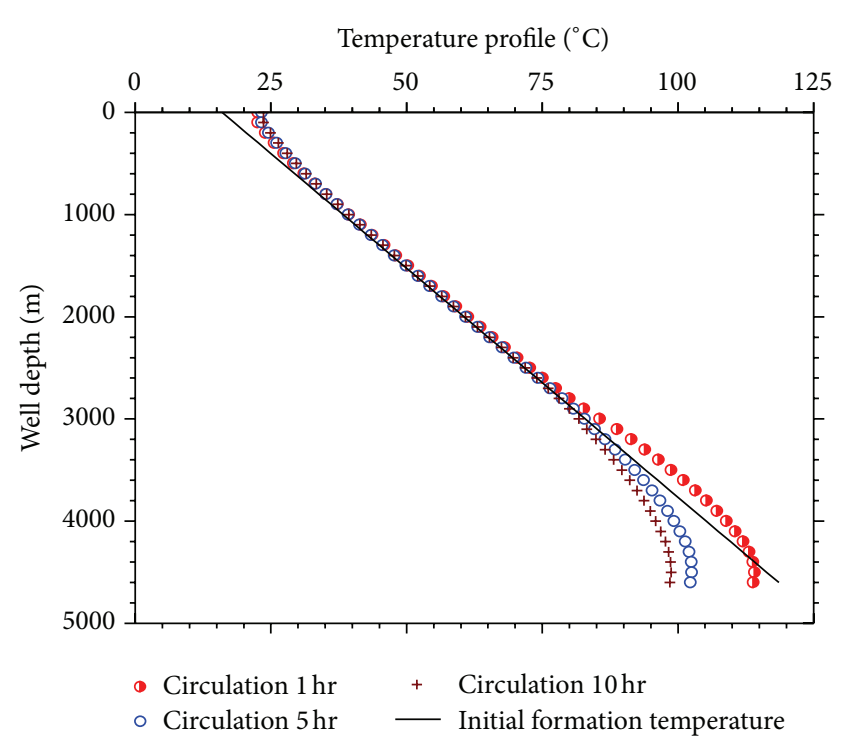

FIGURE 2: Annulus temperature profiles at different circulation time under no lost circulation conditions.

where $T$ is the variable temperature; $\Delta z$ is the step increment in the space coordinate; $n$ is the time node; $i$ indicates the node number in the $r$ direction; $j$ is the node number in the $z$ direction; $a_{i j}, \beta_{i j}, \gamma_{i j}, \delta_{i j}$, and $\lambda_{i j}$ are the matrices of coefficients; SOR is the Gauss-Seidel iterative method if $\omega$ is equal to $1 \mathrm{in}(21)$; the SOR is overrelaxation method if $\omega$ is more than 1; SOR is under relaxation method if $\omega$ is less than 1.

The calculation accuracy depends on the meshing elements and the size of the interval values. In general, it is observed that the vertical element size is less than $3 \%$ of the total well depth to ensure that the annulus temperature profile remains independent of the vertical element size [6].

\section{Model Solution Procedure}

5.1. Basic Data. The basic data of calculation in this study are the referenced literatures reports [6], which are shown in Tables 1, 2, and 3. The flow rate and depth of lost circulation are assumed as $4.01 / \mathrm{s}$ and $3500 \mathrm{~m}$, respectively.

\subsection{Numerical Model Application}

5.2.1. Example Analysis in Circulation Operation Condition. As shown in Figure 2, the annulus temperature distributions as a function of depth at different circulation time under no lost circulation conditions are presented. As intermediate casing depth is $3000 \mathrm{~m}$ (Table 1), the annulus temperatures of cementing section vary under different circulation time, whereas the annulus temperature of open-hole section gradually decreases with the increase of the circulation time. That is because casing thermal conductivity coefficient is 19.4 times than that of formation, resulting in more amount of heat exchange between annulus energy of cementing section and formation compared with that of open hole. Meanwhile, the

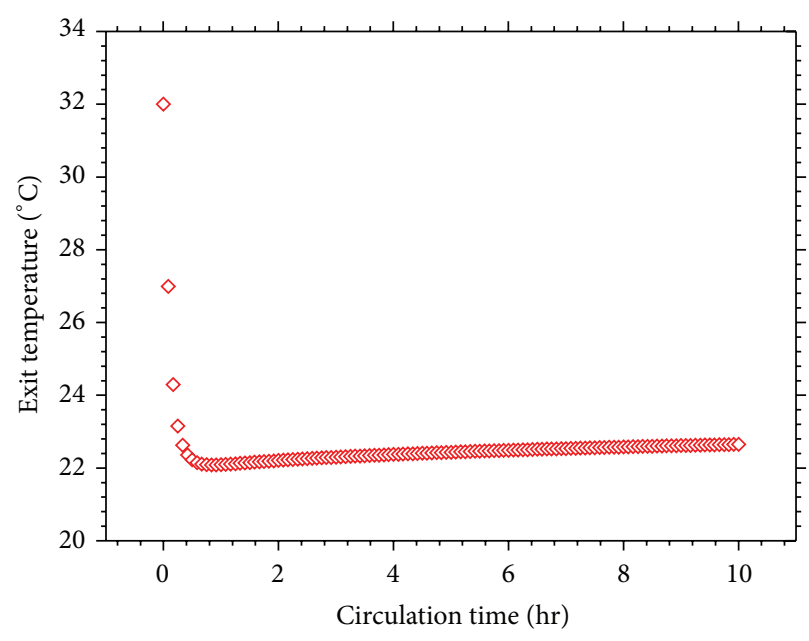

FIgURE 3: Outlet temperature distribution as a function of circulation time under lost circulation condition.

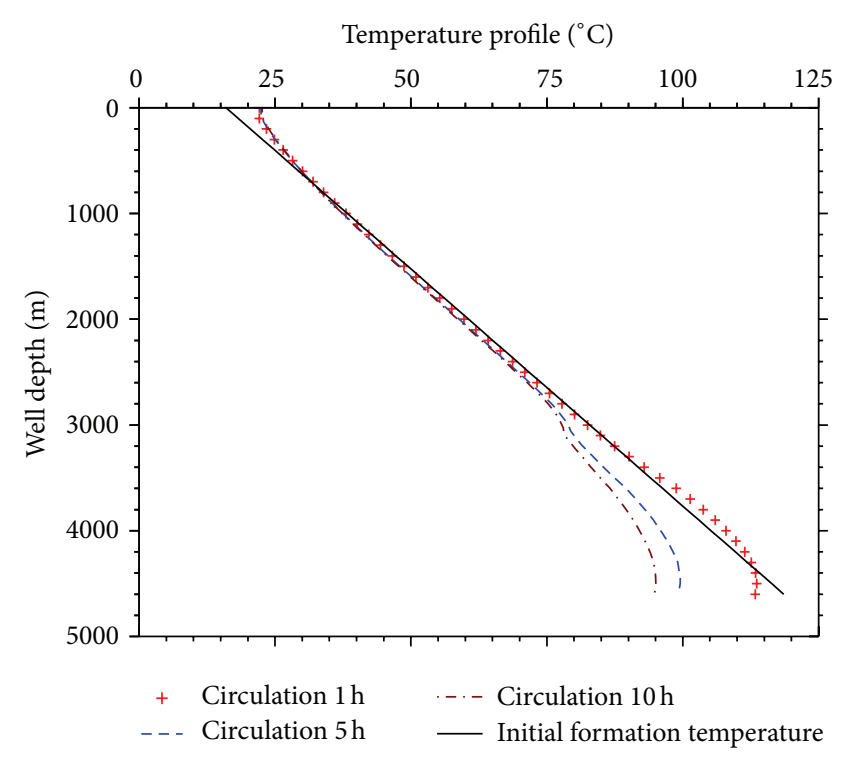

FIGURE 4: Annulus temperature profiles as a function of circulation time under lost circulation conditions.

annulus heat quantity is gradually carried to surface with the increase of the circulation time and thus results in the decrease of annulus temperature of open-hole section [18].

The relationship between outlet temperature and circulation time under lost circulation condition is also investigated. As shown in Figure 3, the outlet temperature rapidly decreases within the initial circulation $(0.5 \mathrm{~h})$ and then gradually increases during the latter circulation. One plausible explanation is that more heat quantity is carried to well mouth at initial circulation compared to that at latter circulation and thus leads to the wellbore wall of well mouth heat.

Under lost circulation conditions, the effect of alterations in circulation time on the annulus temperature distribution is shown in Figure 4. It is found that the annulus temperature of open-hole section continuously decreases as the increase of 
TABLE 1: Basic data of drill string assembly and casing program.

\begin{tabular}{|c|c|c|c|c|c|}
\hline & Drill pipe & Drill collar & First casing & Second casing & Third casing \\
\hline Inner diameter (mm) & 151 & 70 & 486 & 318 & 224 \\
\hline Outer diameter $(\mathrm{mm})$ & 168 & 171 & 508 & 340 & 244 \\
\hline Depth (m) & 4000 & - & 600 & 1500 & 3000 \\
\hline Depth to cement (m) & - & - & 0 & 300 & 1400 \\
\hline
\end{tabular}

TABLE 2: Basic data of thermal physical parameters.

\begin{tabular}{lcccccc}
\hline & Drill pipe/casing & Drill string & Drill fluid & Cement & Formation & Formation fluid \\
\hline Density $\left(\mathrm{kg} / \mathrm{cm}^{3}\right)$ & 8000 & 8900 & 1200 & 2140 & 2640 & 1050 \\
Heat capacity $\left(\mathrm{J} / \mathrm{kg} \cdot{ }^{\circ} \mathrm{C}\right)$ & 400 & 400 & 1600 & 2000 & 800 & 4200 \\
Thermal conductivity $\left(\mathrm{w} / \mathrm{m} \cdot{ }^{\circ} \mathrm{C}\right)$ & 43.75 & 43.75 & 1.75 & 0.70 & 2.25 & 0.50 \\
\hline
\end{tabular}

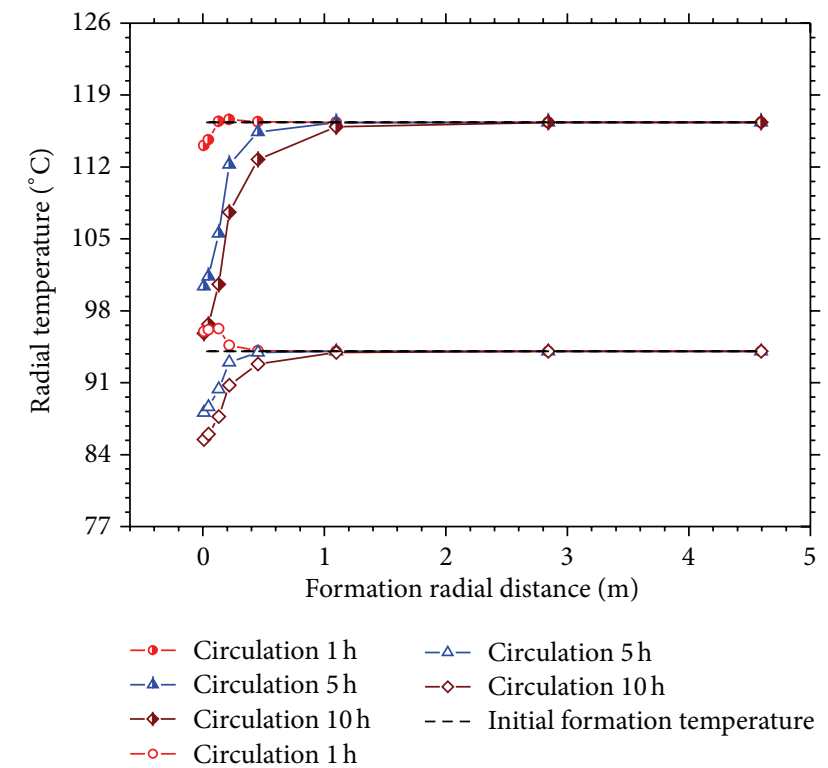

FIGURE 5: Formation radial temperature distributions of lost depth and bottom hole.

the circulation time. Additionally, the closer the bottom hole is, the less decrease the temperature will be, which is in accordance with the result of Figure 2. Meanwhile, the annulus temperature profiles of circulation $5 \mathrm{~h}$ and $10 \mathrm{~h}$ are both lower than the formation temperature below $1500 \mathrm{~m}$. Compared to Figure 2, Figure 4 indicates that the annulus temperature of cement section decreases under lost circulation conditions. The reason is that the annulus fluid temperature at $3500 \mathrm{~m}$ is higher than that of annulus fluid at any depth of cement section. Herein, heat quantity of annulus fluid at $3500 \mathrm{~m}$ flows into the formation increased, which can result in the decrease of the annulus temperature.

Similarly, Figure 5 also reflects the formation radial temperature distributions of lost depth and bottom hole under different circulation time. Noticeably, the formation radial temperature decreases with the increase of the circulation time, whereas the decrease of the surrounding formation temperature at lost depth is less than that of at bottom hole

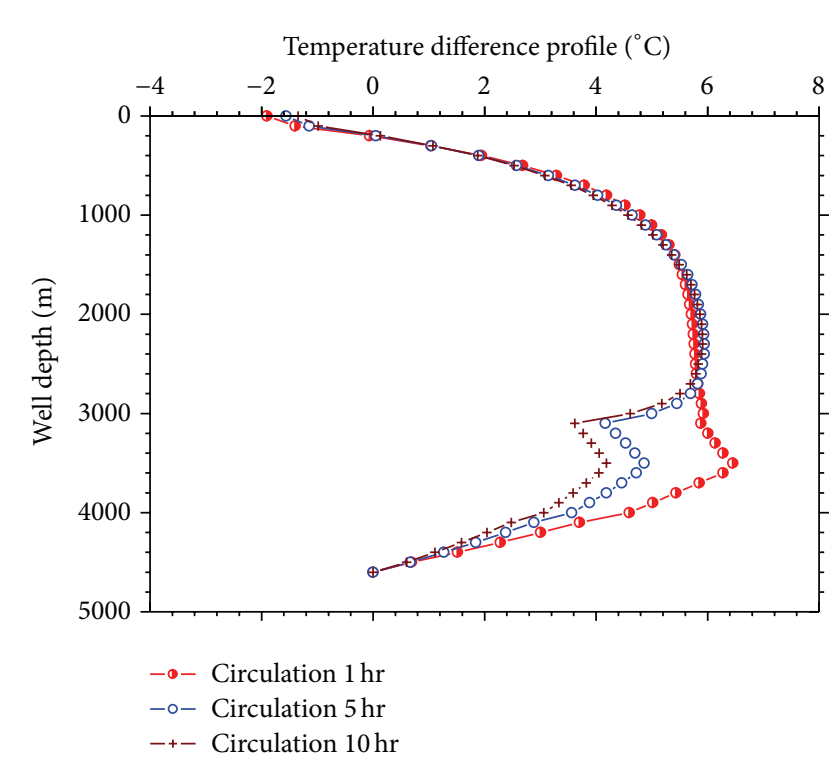

FIGURE 6: Temperature difference profiles between annulus and inside pipe fluid during different circulation time.

during the latter circulation. The surrounding formation is continuously heated by annulus fluid at lost depth during initial circulation stage and then leads to its temperature rise beyond its initial formation temperature as shown in Figure 5 (circulation $1 \mathrm{~h}$ ). Meanwhile, the annulus temperature is lower than formation temperature after long circulation time and thus leads to formation temperature decrease. However, the formation is continuously cooled by circulation fluid at bottom hole and then leads to the temperature of the surrounding formation decrease below the initial formation temperature.

To get a deep insight of the heat transfer mechanism for wellbore during the circulation stage, the temperature difference distribution between annulus and inside pipe fluid under different circulation time is studied. As shown in Figure 6, the temperature difference remarkably decreases from bottom-hole to casing shoe with increasing the circulation time. Meanwhile, the temperature difference changes 
TABLE 3: Other basic data of bottom hole.

\begin{tabular}{lccccc}
\hline Depth $(\mathrm{m})$ & $\begin{array}{c}\text { Total well diameter } \\
(\mathrm{mm})\end{array}$ & $\begin{array}{c}\text { Open hole diameter } \\
(\mathrm{mm})\end{array}$ & Flow rate $(\mathrm{l} / \mathrm{s})$ & $\begin{array}{c}\text { Surface temperature } \\
\left({ }^{\circ} \mathrm{C}\right)\end{array}$ & $\begin{array}{c}\text { Geothermal gradient } \\
\left({ }^{\circ} \mathrm{C} / 100 \mathrm{~m}\right)\end{array}$ \\
\hline 4600 & 660 & 213 & 13.2 & 16 & 2.23 \\
\hline $\begin{array}{l}\text { Inlet temperature } \\
\left({ }^{\circ} \mathrm{C}\right)\end{array}$ & $\begin{array}{c}\text { Outlet temperature } \\
\left({ }^{\circ} \mathrm{C}\right)\end{array}$ & $\begin{array}{c}\text { Plastic viscosity } \\
(\mathrm{mPa} \cdot \mathrm{s})\end{array}$ & $\begin{array}{c}\text { Yield value } \\
(\mathrm{mPa})\end{array}$ & $\begin{array}{c}\text { Consistency } \\
\text { coefficient }\left(\mathrm{mPa} \cdot \mathrm{s}^{n}\right)\end{array}$ & Fluidity point \\
\hline 24 & 32 & 34 & 10 & 0.34 & 0.65 \\
\hline
\end{tabular}

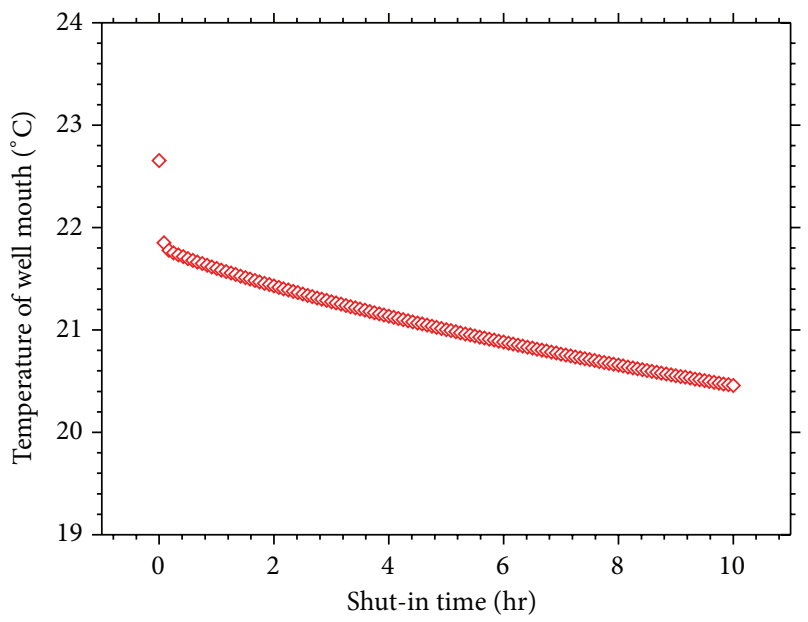

FIGURE 7: Temperature of well mouth during shut-in stage under lost circulation condition.

at the lost circulation point. Additionally, the annulus temperature is higher than the inside pipe fluid temperature in the wellbore except for well mouth section during the whole circulation stage.

5.2.2. Example Analysis in Shut Condition. As shown in Figure 7, the temperature of well mouth continuously decreases during the whole shut-in stage. The result of Figure 3 shows that the outlet temperature increases during the latter circulation, resulting in surrounding formation continuously heated. However, during shut-in stage, as gas is instead of fluid at well mouth, heat exchange between wellbore and formation is less due to heat conductivity of gas. Therefore, the temperature of well mouth gradually decreases with shut-in time increased.

As it is seen from Figure 8, the annulus temperature continuously increases with the increase of shut-in time when the well depth is beyond casing shoe, whereas the annulus temperature hardly varies when the well depth is above the casing shoe point. It spends about $8.5 \mathrm{~h}$ on all fluids of inside pipe and annulus above lost depth flows into formation. Therefore, the heat exchange between the wellbore and formation by heat conduction is more than that of heat convection during $8.5 \mathrm{~h}$ of shut-in. After that, the formation energy fluxes into annulus in the heat conduction way as wellbore fluid is in the static state beyond $8.5 \mathrm{~h}$, thus resulting in the improvement of temperature. Furthermore, the temperature eventually increases to be equal to the formation

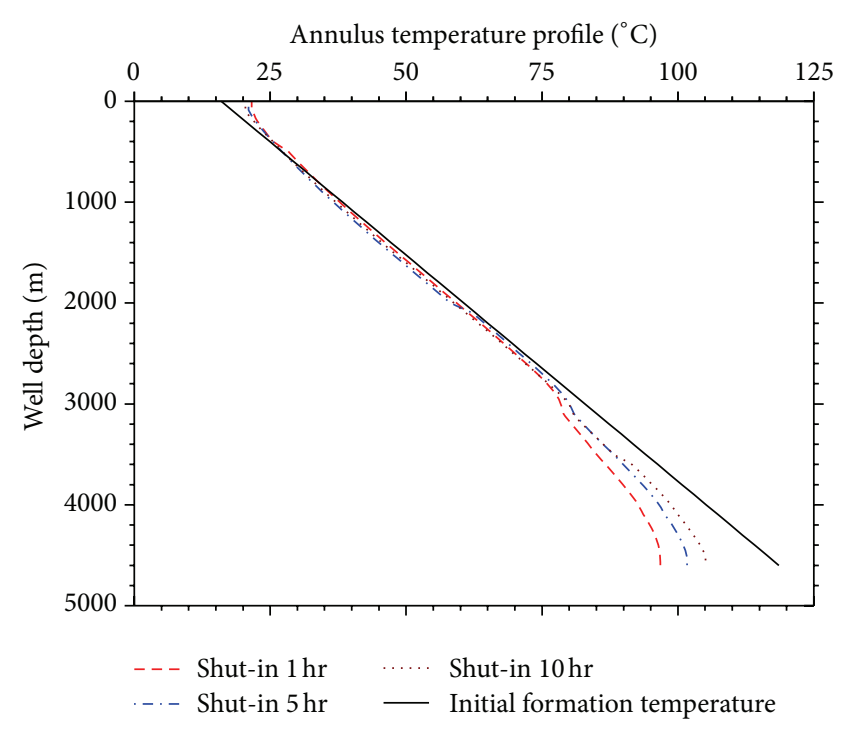

FIGURE 8: Annulus fluid temperature profiles during different shutin time.

temperature. However, when the temperature is above the lost depth, annulus energy which arose from formation is nearly equal to that of the annulus gas transferring to the surrounding formation and the inside pipe drilling fluid by the heat convective way, which leads to the temperature hard change.

Figure 9 indicates that the radial formation temperature decreases with increasing the shut-in time at lost circulation depth and bottom hole, and both of them are lower than that of initial temperature. However, the radial formation temperature at lost depth slowly decreases with the increase of the shut-in time, and temperature change at the bottom hole is larger than that of at lost depth. The reasonable explanation is that the temperature difference between annulus and formation at bottom hole is larger than that of at lost circulation point during circulation stage. Compared to Figure 5, it is surprising that Figure 9 implies that the formation temperature disturbance distance in shut-in stage is larger than that of circulation stage. It is derived from that the starting point of disturbance distance for radial formation temperature is at well wall during the circulation stage, but the starting point of disturbance distance is at inside formation during the shut-in stage which is the destination point of disturbance distance for circulation stage.

As shown in Figure 10, the annulus temperature from top hole $(500 \mathrm{~m})$ to bottom hole is higher than the inside 


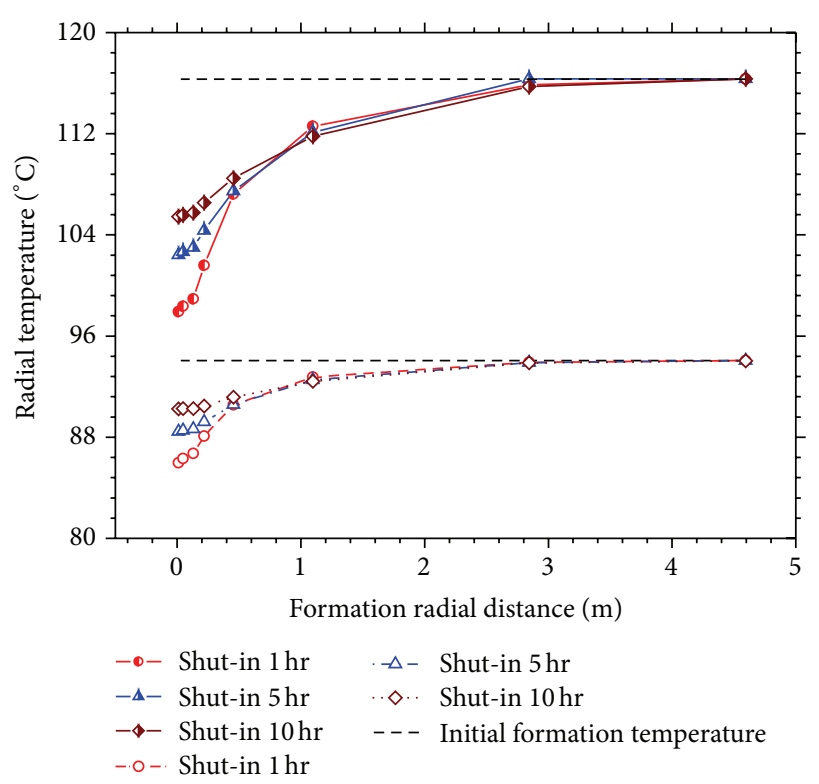

FIGURE 9: Formation radial temperature distributions of lost circulation point and bottom hole during shut-in stage.

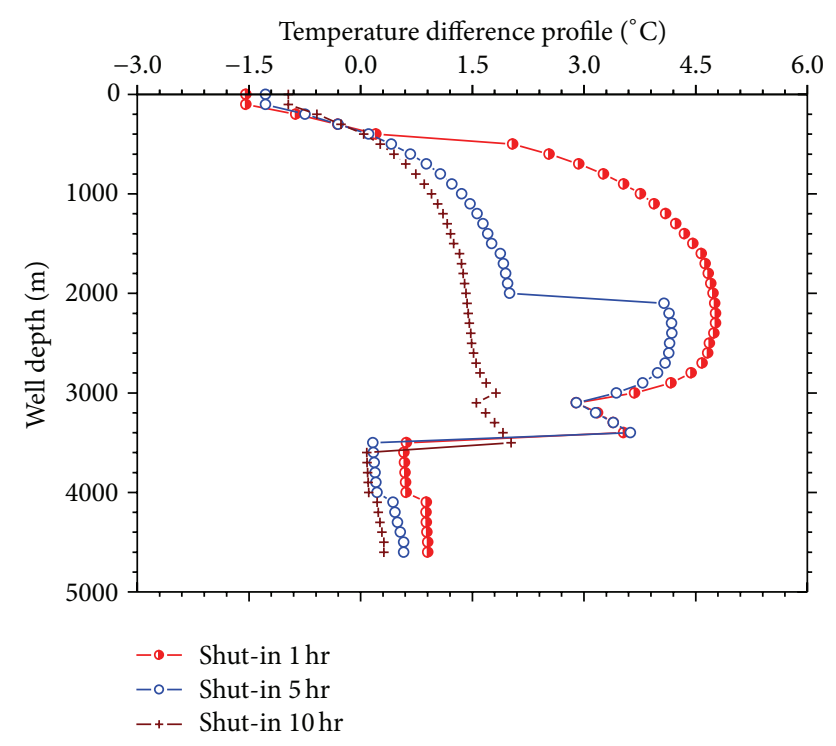

FIGURE 10: Temperature difference profiles between annulus and inside pipe during shut-in stage.

pipe temperature during the whole shut-in stage, and only the temperature difference between annulus and inside pipe from well mouth to the depth of $500 \mathrm{~m}$ is negative value. Furthermore, the temperature difference between annulus and inside pipe gradually decreases with increasing shut-in time and then trends to thermodynamic equilibrium state. From Figure 10, it is observed that the temperature difference between annulus and inside pipe is greatly influenced by the lost depth, make up of string, and casing program.

As shown in Figures 11 and 12, annulus temperature changes of lost depth and bottom hole are related to the

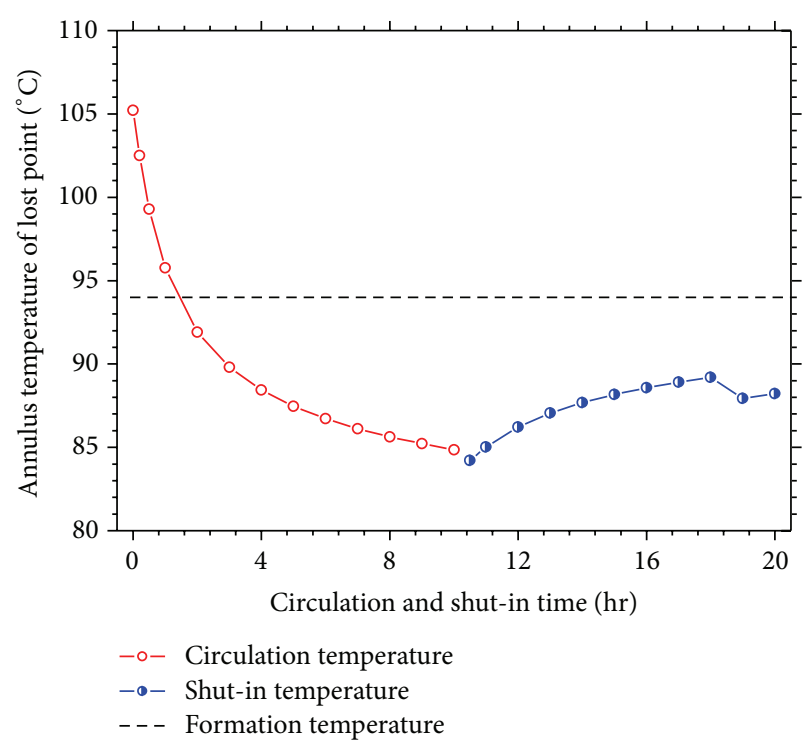

FIGURE 11: Annulus temperature distributions of lost circulation depth during circulation and shut-in stages.

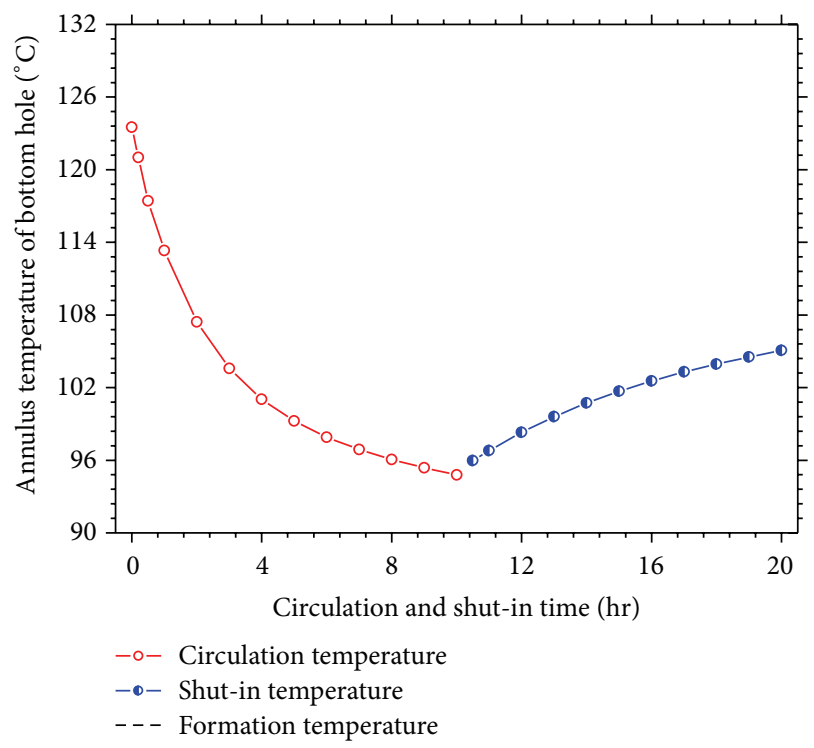

FigURE 12: Annulus temperature distributions of bottom hole during circulation and shut-in stages.

circulation and the shut-in stages. It can be seen that the annulus temperature rapidly decreases during the initial circulation stage and slowly varies in the latter circulation and shut-in stage, followed by the change of annulus temperature showing the same tendency under the two conditions earlier. Meanwhile, the annulus temperatures at initial circulation stage are both higher than that of the initial formation temperature at lost depth and bottom hole, and then both of them are lower than initial formation temperature during latter circulation and shut-in stages. However, before wellbore fluid above the lost depth flows into formation $(8.5 \mathrm{~h})$, it is interestingly noted that the annulus temperature gradually 
increases with the increase of shut-in time at lost depth by heat convection, followed by quickly decreasing, and then slowly increases at bottom hole by heat conduction. Figures 11 and 12 also show that if the annulus temperature after circulation recovers to the initial formation temperature, shut-in time can be longer than that of circulation time [28]. The phenomenon accounts for the reason why long time for shut-in is needed to obtain initial formation temperature.

\section{Discussion}

To demonstrate the applicability of the methodology developed in this work, the OM-16C geothermal well was considered. This well is in Kenya, which was drilled with borehole diameters of $26,17.5,12.25$, and 8.5 in. The casing has 20, $13.375,9.625$, and 7 in diameters at $60,300,750$, and $2680 \mathrm{~m}$ depths, respectively. Temperatures in and around the OM16C geothermal well during circulation and shut-in stages were estimated by the transient heat transfer models. The Horner (Dowdle and Cobb, 1975) and Hasan and Kabir (1994) methods were used to compare our numerical results $[6,12]$.

The input data to simulate the geothermal well are drilling fluid flow rate of $125.3 \mathrm{~m}^{3} / \mathrm{h}$, surface temperature of $21.6^{\circ} \mathrm{C}$, and drilling fluid properties which include the thermal conductivity of $0.85 \mathrm{~W} / \mathrm{m} \cdot{ }^{\circ} \mathrm{C}$, the density of $1100 \mathrm{~kg} / \mathrm{m}^{3}$, the viscosity of $0.052 \mathrm{~Pa} . \mathrm{s}$, and specific heat of $1200 \mathrm{~J} / \mathrm{kg} .{ }^{\circ} \mathrm{C}$. Circulation time was $16 \mathrm{~h}$.

A compilation of main results obtained in these thermal studies was presented in Figure 13. We reckoned that the logged temperature of shut-in 27 days was approximately equal to the static formation temperature (SFT) due to thermal recovery conditions during the long time shut-in. As shown in Figure 13, it can be observed that the measured temperature was satisfactorily matched with the simulated temperature (continuous line). Figure 13 also showed the SFT calculated by means of Horner (Dowdle and Cobb, 1975) and Hasan and Kabir (1994) methods [6,12]. Obviously, as shown in Figure 13, the Hasan-Kabir method is closer to the SFT compared to the Horner method. Differences between the computed data (or simulated 27 days) and measured values were estimated and expressed as a percentage deviation based on the result of Figure 13, and the percentage deviation between the simulated SFT and analytical methods as also computed from Figure 13. It can be observed the maximum deviation between measured and simulated data is 3.1\% and minimum deviation is $2.24 \%$, which corresponded to the control error in engineering. The best approximation to the simulated SFT values corresponded to the Hasan-Kabir, which presented minimum differences of $3.50 \%, 4.12 \%$, and $4.08 \%$. Therefore, the simulated SFT method in this work is better than that of the analytical methods (Horner and Hasan and Kabir).

\section{Conclusions}

In this study, a set of numerical models have been developed to study the transient heat transfer processes which occurs

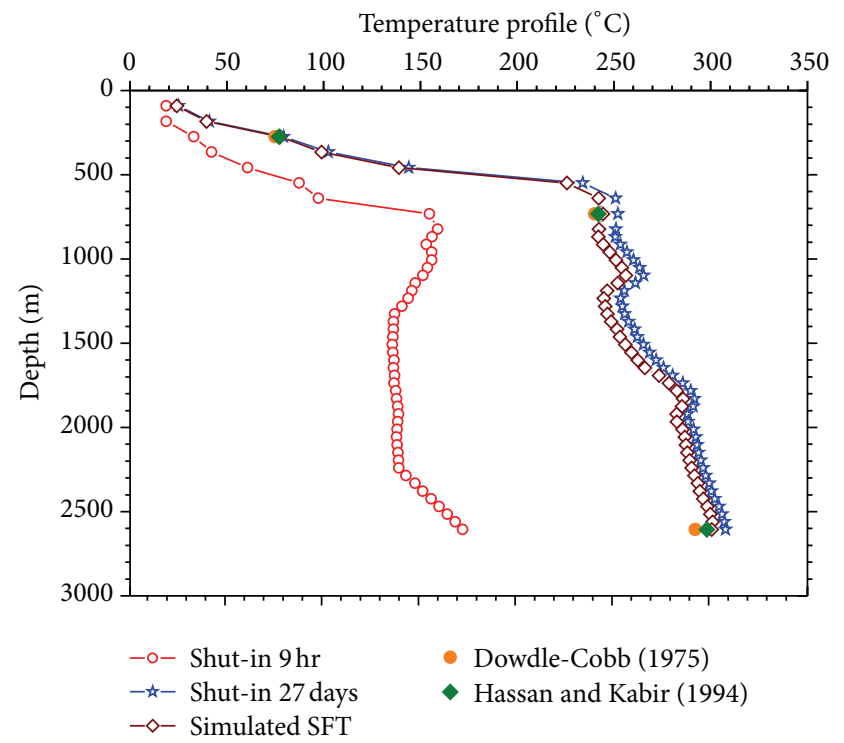

FIGURE 13: Simulated and logged temperature profiles in OM16C geothermal well during shut-in stages. Also shown the SFTs estimated with the Horner (Dowdle and Cobb, 1975) and Hasan and Kabir (1994) methods and with this work $[6,12]$.

in oil-gas or geothermal well during circulation and shutin stages under lost circulation conditions. The equations properly account for the energy conservation in each region of a well, and mass balances are performed at any numerical node where annulus fluid fluxes into formation. Heat transfer coefficients and thermophysical properties (gas instead of fluid) in the annulus and the surrounding formation change due to lost circulation. Simulation results show that the temperature distributing characters of the wellbore and surrounding formation are remarkably influenced by the lost depth and casing program during the whole circulation and shut-in stage. Additionally, the disturbance distance of formation temperature at shut-in stage is larger than that of at circulation stage at the same time. Moreover, it is necessary to prolong shut-in time than circulation time in order to obtain accurate initial formation temperature. The present work can provide a new way to improve the present methodology.

\section{Acknowledgments}

The authors appreciate the financial support by China National Natural Science Foundation (no. 51134004; 51104124; 51204142; 51204140), Major State Science and Technology Special Project of China (no. 2011ZX05021-003), Ph.D. Programs Foundation of Ministry of Education of China (no. 20125121110001), and Southwest Petroleum University of Young Scientific Research Innovation Team Foundation (no. 2012XJZT003). The authors would also like to appreciate their laboratory members for the generous help.

\section{References}

[1] S. Fomin, V. Chugunov, and T. Hashida, "Analytical modelling of the formation temperature stabilization during the borehole 
shut-in period," Geophysical Journal International, vol. 155, no. 2, pp. 469-478, 2003.

[2] S. Fomin, T. Hashida, V. Chugunov, and A. V. Kuznetsov, "A borehole temperature during drilling in a fractured rock formation," International Journal of Heat and Mass Transfer, vol. 48, no. 2, pp. 385-394, 2005.

[3] A. Bassam, E. Santoyo, J. Andaverde, J. A. Hernández, and O. M. Espinoza-Ojeda, "Estimation of static formation temperatures in geothermal wells by using an artificial neural network approach," Computers and Geosciences, vol. 36, no. 9, pp. 11911199, 2010.

[4] A. Garcia, I. Hernandez, G. Espinosa, and E. Santoyo, “TEMLOPI: a thermal simulator for estimation of drilling mud and formation temperatures during drilling of geothermal wells," Computers and Geosciences, vol. 24, no. 5, pp. 465-477, 1998.

[5] G. Espinosa-Paredes, A. Garcia, E. Santoyo, and I. Hernandez, "TEMLOPI/V.2: a computer program for estimation of fully transient temperatures in geothermal wells during circulation and shut-in," Computers and Geosciences, vol. 27, no. 3, pp. 327$344,2001$.

[6] W. L. Dowdle and W. M. Cobb, "Static formation temperature from well logs-an empirical method," Journal of Petroleum Technology, vol. 27, no. 11, pp. 1326-1330, 1975.

[7] J. L. G. Santander, P. Castańeda Porras, J. M. Isidro, and P. Fernández de Córdoba, "Calculation of some integrals arising in heat transfer in geothermics," Mathematical Problems in Engineering, vol. 2010, Article ID 784794, 13 pages, 2010.

[8] I. M. Kutasov, "Dimensionless temperature, cumulative heat flow and heat flow rate for a well with a constant bore-face temperature," Geothermics, vol. 16, no. 5-6, pp. 467-472, 1987.

[9] I. M. Kutasov and L. V. Eppelbaum, "Prediction of formation temperatures in permafrost regions from temperature logs in deep wells-field cases," Permafrost and Periglacial Processes, vol. 14, no. 3, pp. 247-258, 2003.

[10] I. M. Kutasov and L. V. Eppelbaum, "Determination of formation temperature from bottom-hole temperature logs-a generalized Horner method," Journal of Geophysics and Engineering, vol. 2, no. 2, pp. 90-96, 2005.

[11] X. C. Song and Z. C. Guan, "Coupled modeling circulating temperature and pressure of gas-liquid two phase flow in deep water wells," Journal of Petroleum Science and Engineering, vol. 92-93, pp. 124-131, 2012.

[12] A. R. Hasan and C. S. Kabir, "Static reservoir temperature determination from transient data after mud circulation," $S P E$ Drilling and Completion, vol. 9, no. 1, pp. 17-24, 1994.

[13] F. Ascencio, A. García, J. Rivera, and V. Arellano, "Estimation of undisturbed formation temperatures under spherical-radial heat flow conditions," Geothermics, vol. 23, no. 4, pp. 317-326, 1994.

[14] E. Santoyo, Transient numerical simulation of heat transfer processes during drilling of geothermal wells [Ph.D. thesis], University of Salford, Salford, UK, 1998.

[15] L. R. Raymond, "Temperature distribution in a circulating drilling fluid," Journal of Petroleum Technology, vol. 21, no. 3, pp. 333-341, 1969.

[16] G. R. Wooley, "Computing dowhole temperatures in circulation, injection and production wells," Journal of Petroleum Technology, vol. 32, no. 9, pp. 1509-1522, 1980.

[17] R. M. Beirute, "A circulating and shut-in well-temperatureprofile simulator," Journal of Petroleum Technology, vol. 43, no. 9, pp. 1140-1146, 1991.
[18] G. Espinosa-Paredes and A. Garcia-Gutierrez, "Thermal behaviour of geothermal wells using mud and air-water mixtures as drilling fluids," Energy Conversion and Management, vol. 45, no. 9-10, pp. 1513-1527, 2004.

[19] O. García-Valladares, P. Sánchez-Upton, and E. Santoyo, "Numerical modeling of flow processes inside geothermal wells: an approach for predicting production characteristics with uncertainties," Energy Conversion and Management, vol. 47, no. 11-12, pp. 1621-1643, 2006.

[20] M. N. Luheshi, "Estimation of formation temperature from borehole measurements," Geophysical Journal of the Royal Astronomical Society, vol. 74, no. 3, pp. 747-776, 1983.

[21] A. García, E. Santoyo, G. Espinosa, I. Hernández, and H. Gutiérrez, "Estimation of temperatures in geothermal wells during circulation and shut-in in the presence of lost circulation," Transport in Porous Media, vol. 33, no. 1-2, pp. 103-127, 1998.

[22] G. Espinosa-Paredes, A. Morales-Díaz, U. Olea-González, and J. J. Ambriz-Garcia, "Application of a proportional-integral control for the estimation of static formation temperatures in oil wells," Marine and Petroleum Geology, vol. 26, no. 2, pp. 259268, 2009.

[23] G. Espinosa-Paredes and E. G. Espinosa-Martínez, "A feedbackbased inverse heat transfer method to estimate unperturbed temperatures in wellbores," Energy Conversion and Management, vol. 50, no. 1, pp. 140-148, 2009.

[24] H. H. Keller, E. J. Couch, and P. M. Berry, "Temperature distribution in circulating mud columns," Journal of the Society of Petroleum Engineering, vol. 13, no. 1, pp. 23-30, 1973.

[25] M. C. Zhong and R. J. Novotny, "Accurate prediction wellbore transient temperature profile under multiple temperature gradients: finite difference approach and case history," in Proceedings of the SPE Annual Technical Conference and Exhibition, pp. 5389-5401, Denver, Colo, USA, October 2003.

[26] K.-L. Hsiao, "Viscoelastic fluid over a stretching sheet with electromagnetic effects and nonuniform heat source/sink," Mathematical Problems in Engineering, vol. 2010, Article ID 740943, 14 pages, 2010.

[27] D. W. Marshall and R. G. Bentsen, "A computer model to determine the temperature distributions in a wellbore," Journal of Canadian Petroleum Technology, vol. 21, no. 1, pp. 63-75, 1982.

[28] L. V. Eppelbaum and I. M. Kutasov, "Determination of the formation temperature from shut-in logs: estimation of the radius of thermal influence," Journal of Applied Geophysics, vol. 73, no. 3, pp. 278-282, 2011. 


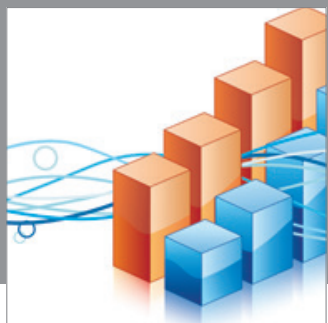

Advances in

Operations Research

mansans

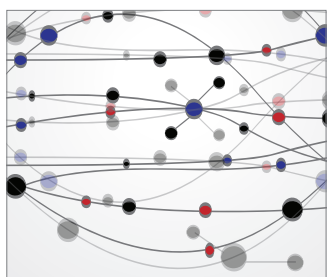

The Scientific World Journal
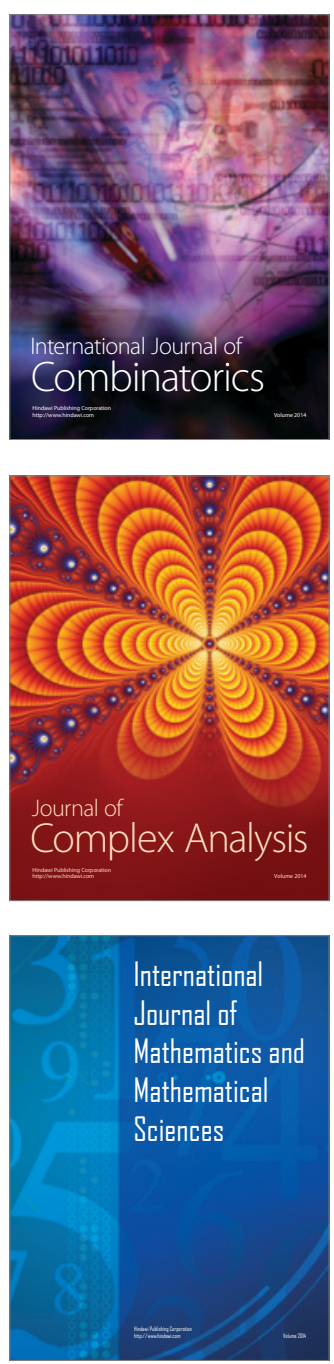
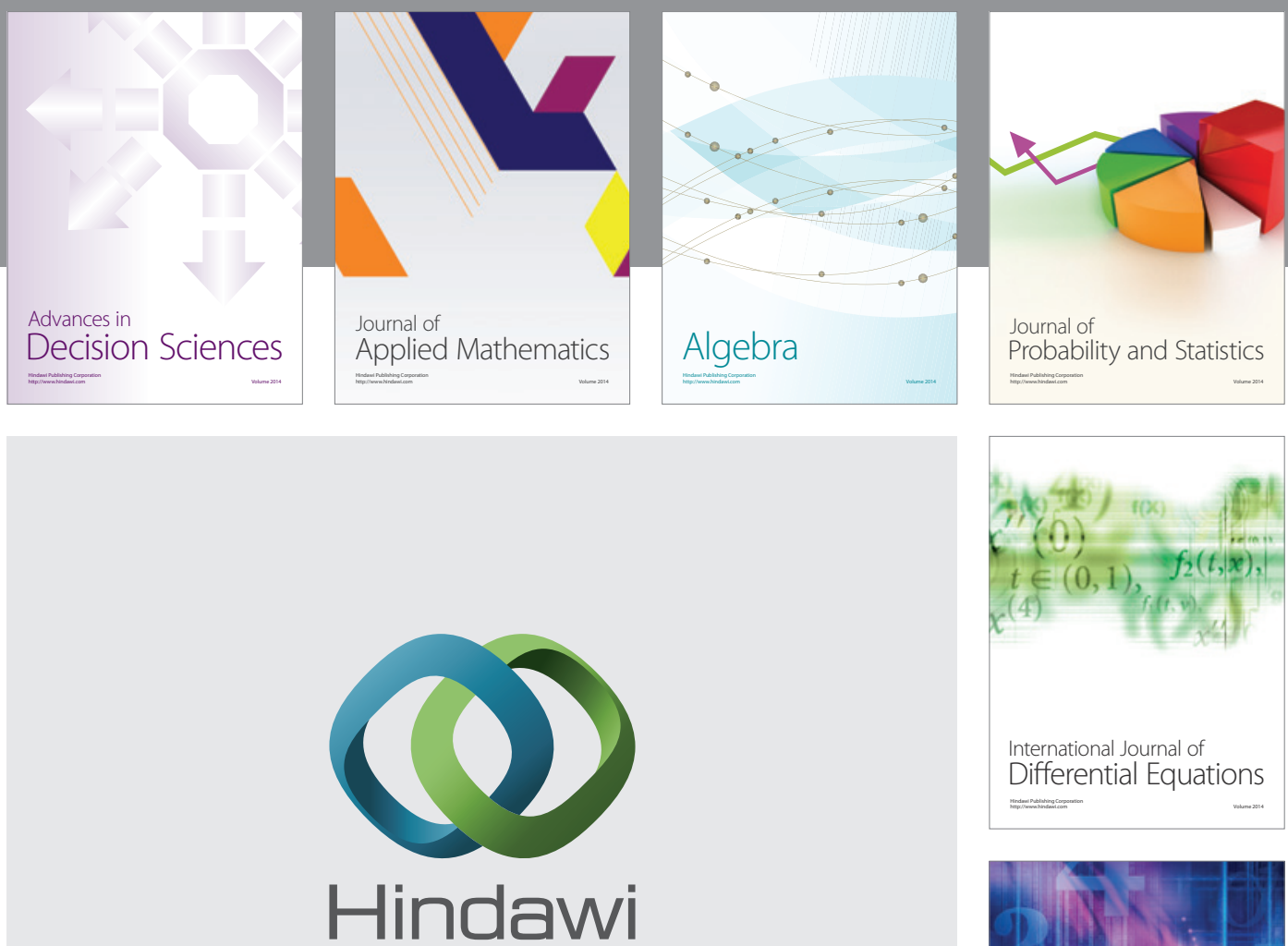

Submit your manuscripts at http://www.hindawi.com
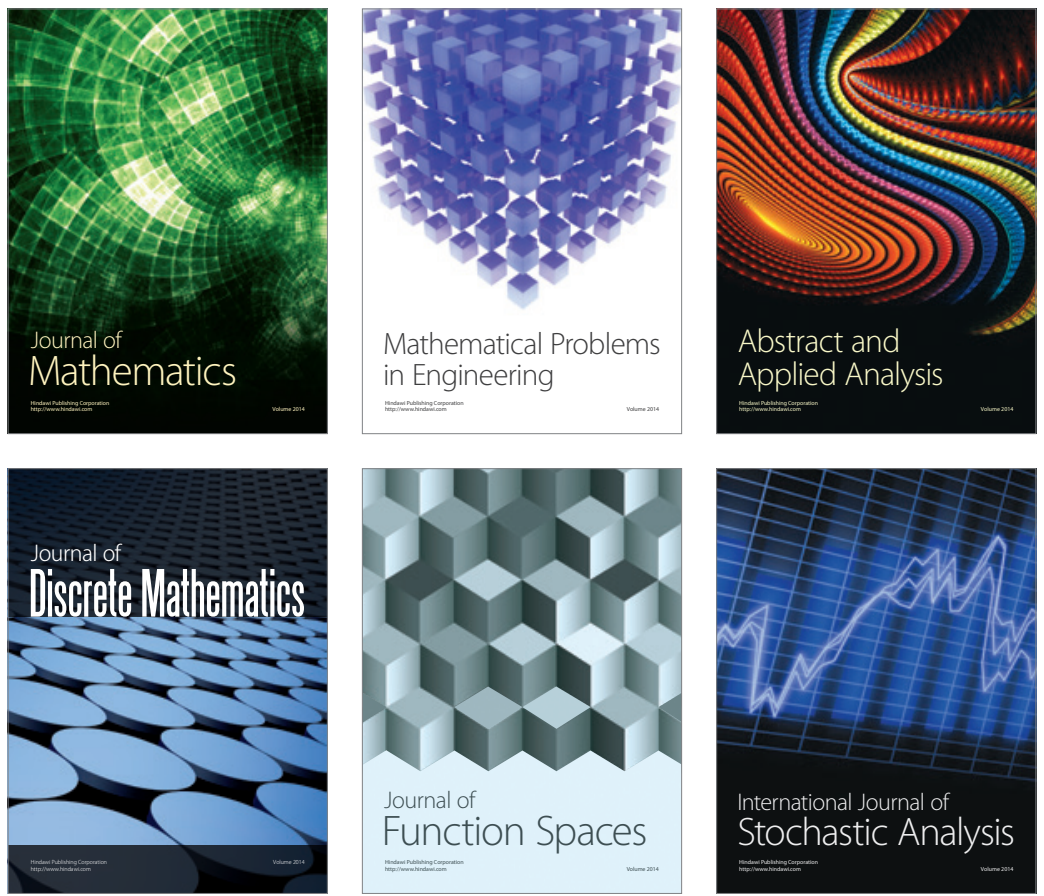

Journal of

Function Spaces

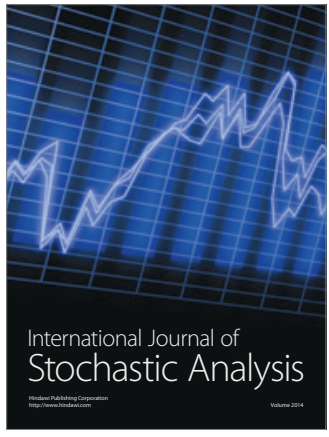

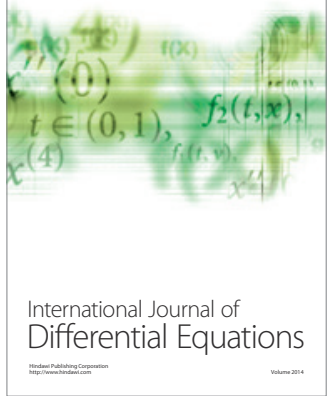
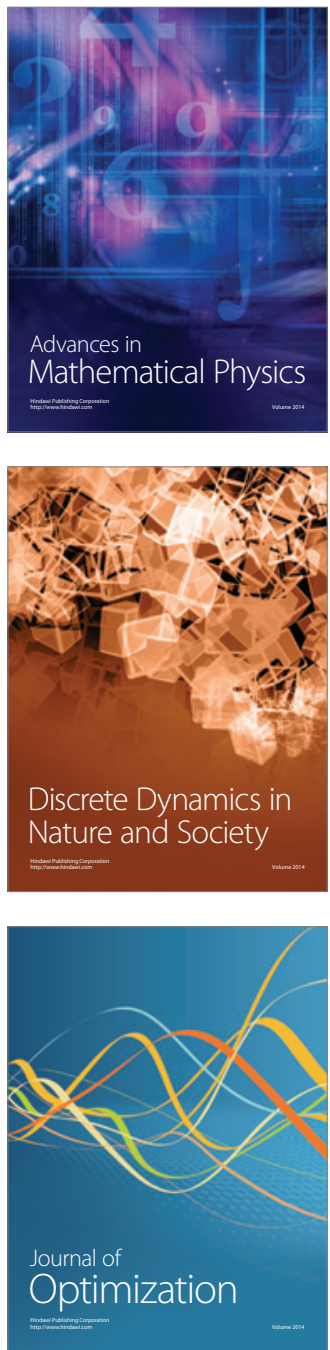